\title{
openheart Early reduction of left atrial function predicts adverse clinical outcomes in patients with severe aortic stenosis undergoing transcatheter aortic valve replacement
}

Jolanda Sabatino, ${ }^{1}$ Salvatore De Rosa (1) ${ }^{1}$ Isabella Leo, ${ }^{1}$ Antonio Strangio, ${ }^{1}$ Sabrina La Bella, ${ }^{1}$ Sabato Sorrentino, ${ }^{1}$ Annalisa Mongiardo, ${ }^{1}$ Carmen Spaccarotella, ${ }^{1}$ Alberto Polimeni, ${ }^{1}$ Ciro Indolfi ${ }^{1,2}$

To cite: Sabatino J, De Rosa S, Leo I, et al. Early reduction of left atrial function predicts adverse clinical outcomes in patients with severe aortic stenosis undergoing transcatheter aortic valve replacement. Open Heart 2021;8:e001685. doi:10.1136/ openhrt-2021-001685

Received 12 April 2021 Accepted 18 June 2021

Check for updates

(c) Author(s) (or their employer(s)) 2021. Re-use permitted under CC BY-NC. No commercial re-use. See rights and permissions. Published by BMJ.

${ }^{1}$ Department of Medical and Surgical Sciences, Magna Graecia University of Catanzaro, Catanzaro, Italy

${ }^{2}$ Mediterranea Cardiocentro, Napoli, Italy

Correspondence to Professor Salvatore De Rosa; saderosa@unicz.it

Professor Ciro Indolfi; indolfi@ unicz.it

\section{ABSTRACT}

Aims To investigate the changes in left atrial strain (LAS) after correction of severe aortic stenosis (AS) with transcatheter aortic valve replacement (TAVR) and assess its prognostic impact.

Methods and results One hundred consecutive patients with severe symptomatic AS who underwent TAVR at the Magna Graecia University of Catanzaro underwent echocardiographic examination including assessment of LAS before and after TAVR. Independent investigators collected outcome data and information. The primary study outcome was the difference in $\triangle$ LAS (postTAVR-preTAVR) between patients those met the main clinical endpoint (a composite of cardiovascular mortality and heart failure hospitalisation) and those not meeting the endpoint. During a median follow-up of 31 months, 35 patients $(35 \%)$ met the combined clinical endpoint. The difference between LAS post-TAVR and LAS pre-TAVR ( $\triangle$ LAS) was significantly larger in patients who met the combined endpoint ( $H R=0.76(0.67-0.86) ; p<0.001)$. Multivariate logistic regression analysis including $\triangle \mathrm{LAS}$, EuroSCORE II and left ventricular ejection fraction showed that $\triangle \mathrm{LAS}$ $(H R=0.80, p<0.001)$ was the only independent predictor of the combined clinical endpoint. Finally, a Kaplan-Maier analysis showed that patients with a $\triangle \mathrm{LAS}$ above its median value had a significantly better event-free survival compared with those below the median $(p<0.001)$. Conclusions A lower reduction in $\triangle \mathrm{LAS}$ after TAVR was an independent predictor of the primary composite outcome of cardiovascular death and hospitalisation for heart failure.

\section{BACKGROUND}

Left ventricular (LV) systolic function is a known prognostic marker in cardiovascular disease. ${ }^{12}$ Much less evidence is available about the role of left atrial (LA) function, despite some studies showed how an early functional impairment is often evident before LA dilatation becomes manifest. ${ }^{3-5}$

\section{Key questions}

What is already known about this subject?

- Successful removal of left ventricular pressure afterload has been revealed to normalise left atria size.

- Left atrial strain (LAS) is emerging as a more precise and reliable index of atrial function.

What does this study add?

- Improvement in LAS after transcatheter aortic valve replacement (TAVR) is an independent predictor of adverse clinical outcomes.

How might this impact on clinical practice?

- If confirmed in larger studies, our results pave the way to the use of LAS and its change after TAVR as a clinical biomarker to guide the clinical management of these patients.

The left atrium has a key role in modulating LV filling through its functions of reservoir, conduit and booster pump. ${ }^{6}$ Several studies have demonstrated the capacity of twodimensional (2D) speckle-tracking echocardiography (STE) to assess left atrial function in adult patients and in children. ${ }^{7-14}$

Recently, 2D LA strain has been proposed to accurately categorise diastolic function in adults. ${ }^{15}$ Furthermore, it has been observed that LA function is associated with adverse long-term outcomes in different cardiomyopathies as well as in patients with aortic stenosis (AS). ${ }^{16-21}$ Treatment of degenerative AS has become increasingly challenging with the constant increase in mean age at diagnosis. ${ }^{22}$ Transcatheter aortic valve replacement (TAVR), in the last decade, has emerged as a promising alternative to surgical aortic valve replacement (AVR) for selected patients. To 
the best of our knowledge, no data are available to date on the prognostic value of LA strain (LAS) measured by means of 2D STE after TAVR.

We investigated the change in LAS in patients with severe AS undergoing TAVR and its prognostic.

\section{METHODS}

Consecutive patients with severe symptomatic AS who underwent TAVR at the Magna Graecia University of Catanzaro from December 2016 to February 2020 were screened. The echocardiographic examinations were carried out as part of routine clinical care. Patients were included if echocardiographic movie clips, before and after the procedure (pre-TAVR and post-TAVR), were available for LA 2D STE analysis, in addition to conventional echo-Doppler parameters. Exclusion criteria were appointed as follows: presence of a pacemaker, concomitant clinically relevant valvular disease (at least moderate stenosis or valvular regurgitation, $n=5$ ), atrial fibrillation at the time of the examination $(n=3)$, poor echogenicity or poor image quality $(\mathrm{n}=3)$.

Demographic and laboratory data, comorbidities, as well as functional status, were assessed in all patients. Patients were followed up for the occurrence of adverse events by means of clinical visits.

The investigation conforms with the principles outlined in the Declaration of Helsinki. All patients gave informed consent to the study.

\section{Transthoracic Doppler echocardiography \\ Standard echocardiography}

Transthoracic echocardiographic examinations were performed by using a commercially available ultrasound system (Vivid E95 system, General Electric, Horton, Norway) equipped with a M5S adult transducer, according to the standardised protocol of the Magna Graecia University of Catanzaro. ${ }^{23}$ Raw data were transferred to a commercially available workstation (EchoPAC V.112.99, Research Release, GE Healthcare) and analysed offline.

The continuity equation was used to measure aortic valve area, according to current guidelines, ${ }^{24}$ and indexed to body surface area. The peak aortic jet velocity was assessed with continuous-wave Doppler from multiple acoustic views and transaortic mean pressure gradient was obtained according to the Bernoulli equation.

The time-velocity integral of the $\mathrm{LV}$ outflow tract (LVOT) was calculated in apical five-chamber view. Stroke volume was determined with LVOT area estimated from the parasternal long-axis view in systole, at the base of the valve leaflets. ${ }^{25}$

Chamber size and function were assessed as previously described according to the latest guidelines. ${ }^{26}$ Values for LV diameters and volumes were adjusted for body surface area (BSA). ${ }^{26}$ Left ventricular ejection fraction (LVEF) was calculated according to the modified Simpson's rule, while left ventricular mass index was adjusted

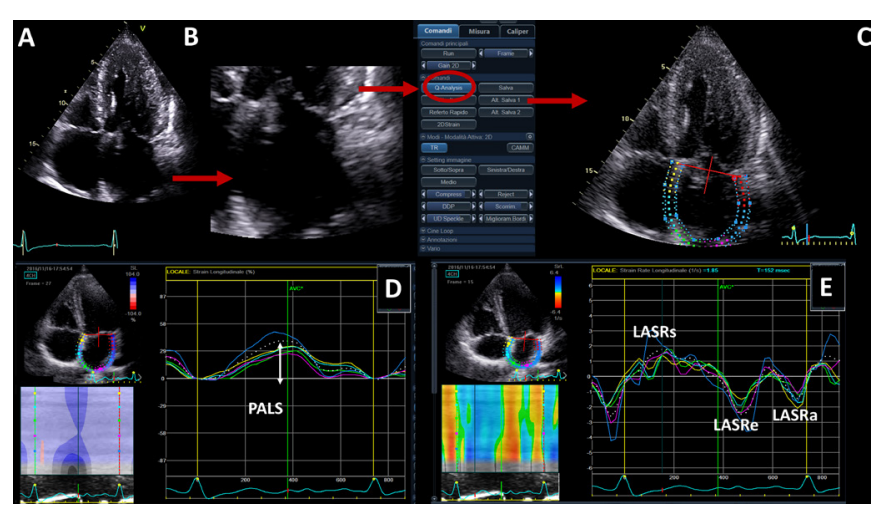

Figure 1 How to assess left atrial strain. Acquire a fourchamber apical view (A). Adjust the image sector in order to focus on the left atrium (B). After recording the images (from three to five cycles) select on the EchoPAC offline platform the $\mathrm{Q}$ analysis option. Trace the atrial border (C). Approve and process the curve analysis, generating the peak atrial longitudinal strain (PALS, white arrow, D) and strain rate (left atrial peak strain rate S-wave (LASRs); left atrial strain rate E-wave (LASRe); left atrial strain rate A-wave (LASRa)).

according to the formula of the American Society of Echocardiography.

The following standard parameters were measured by one expert cardiologist, according to the latest guidelines $^{27}$ : mitral inflow early-to-late diastolic flow ratio, mitral lateral and septal peak early diastolic tissue velocities, mitral E-to-mean E' ratio (E/E'). Moreover, left atrial volume indexed (LAVi) to BSA was derived from the apical four chamber view. The change in LAVi after TAVR compared with baseline was calculated as the delta value $(\triangle \mathrm{LAVi})$.

The maximum tricuspid regurgitation velocity was used to estimate the peak tricuspid regurgitation gradient by means of the modified Bernoulli equation. The pulmonary artery systolic pressure (PASP) was measured from this by adding right atrial pressure (estimated by looking
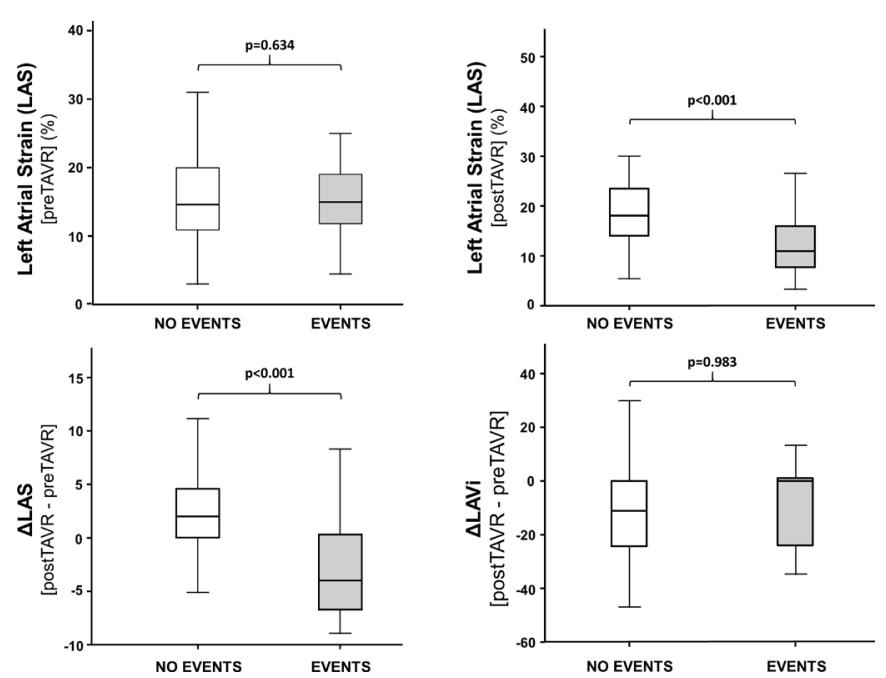

Figure 2 Left atrial parameters according to patients' outcome post-TAVR. LAS, left atrial strain; LAVi, left atrial volume index; TAVR, transcatheter aortic value replacement. 


\section{$\Delta$ LAS}

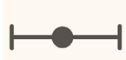

LVEF

\section{EuroSCORE II}

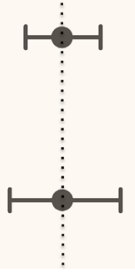

$1.00(0.93-1.06) p=0.755$

Figure 3 Multivariate logistic regression analysis showing that $\triangle \mathrm{LAS}(\mathrm{HR}=0.80, \mathrm{p}<0.001)$ was an independent predictor of the main clinical endpoint. LAS, left atrial strain; LVEF, left ventricular ejection fraction.

at diameter and respiratory variation of inferior vena cava).

\section{Two-dimensional speckle-tracking analysis}

Two-dimensional STE analyses were performed by a different experienced cardiologist, using EchoPAC software.

For speckle-tracking analysis, apical four-chamber views images were acquired, using standard 2D grey scale echocardiography, during breath hold and with a stable ECG recording. ${ }^{14} 23$

To assure optimal tracking, three consecutive heart cycles were collected at a frame rate of 50-80 frames/s, sinus rhythm and $\leq 10 \%$ variability in heart rate. ${ }^{1423}$

As depicted in figure 1, the LA endocardial border was traced excluding appendage and pulmonary veins from

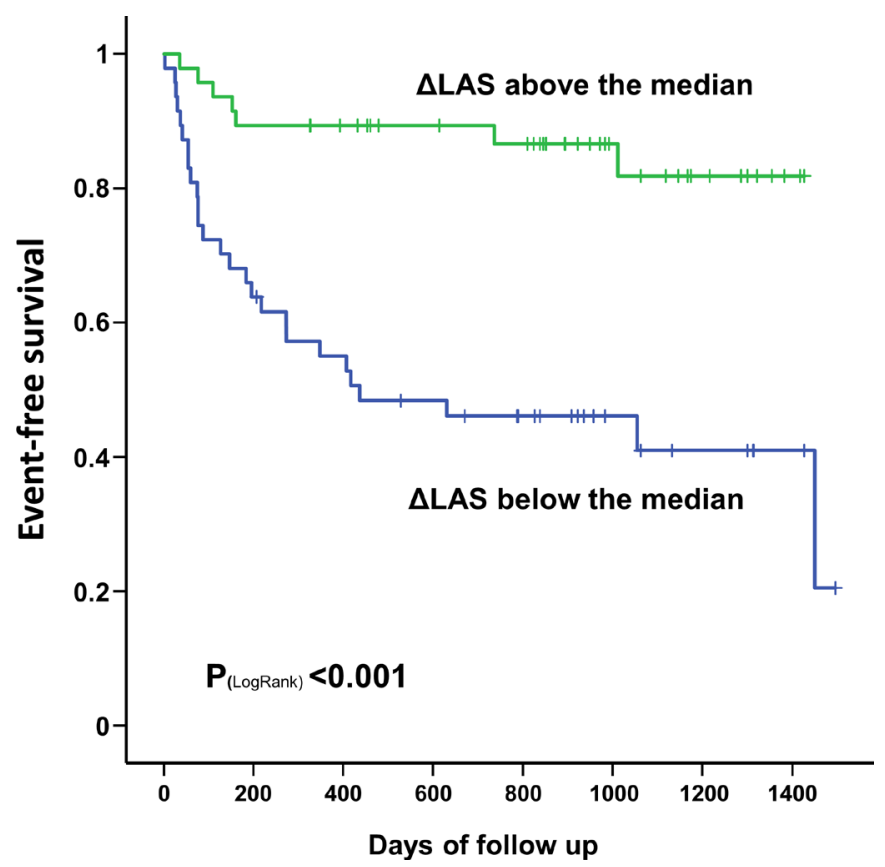

Figure 4 Kaplan-Meier curve displaying the significant difference of event-free survival between patients with a $\triangle \mathrm{LAS}$ above its median value compared with those below the median $(p<0.001)$. LAS, left atrial strain. the LA cavity, from the apical four-chamber view. Hence, LA longitudinal strain and strain rate curves were created from the software throughout the cardiac cycle. The LA wall longitudinal lengthening during the LV systole was measured and employed for the analysis (LA peak systolic strain, LAS). ${ }^{14}$ The change in LAS after TAVR compared with baseline was calculated as the delta value $(\triangle \mathrm{LAS})$.

\section{TAVR}

TAVR was carried out in all population in accordance with standard technique. ${ }^{20} \mathrm{~A}$ transfemoral vascular access was adopted in all patients. Both self-expandable and balloon-expandable prosthetic valves were employed.

\section{Follow-up}

Two independent investigators, blinded to the analyses results, collected outcome data and information.

Clinical data were acquired in all population through telephone contact with the patients, with the referring physician or through an outpatient medical visit. Hospital records were employed to corroborate outcomes.

The main clinical endpoint was the composite of cardiovascular-mortality and heart failure hospitalisation. Secondary endpoints included: death for any cause, new myocardial infarction and stroke/transient ischaemic attack.

AS patients were divided in two comparative groups, those who meet the primary endpoint (cardiovascular death or hospitalisation for heart failure) were labelled as 'EVENTS', while all others as 'NO EVENTS'.

\section{Reproducibility study}

Interobserver variability for atrial deformation properties was assessed in 20 randomly selected examinations and was calculated as the ratio (indicated as a percentage) of the difference between the measures obtained by each investigator (indicated as an absolute value) divided by the mean of the two measures, and as intraclass correlation coefficients. Intraobserver variability was assessed by a similar approach.

\section{Statistical analysis}

Statistical analyses were performed using JASP (V.0.14.1) and KMWin (V.1.52). Distribution of variables was judged by visual inspection of frequency histograms, and normality was proven using the Shapiro-Wilk test. Continuous variables were presented as mean and SD and were compared through the unpaired Student's t-test or the Mann-Whitney $\mathrm{U}$ test in case of a non-normal distribution. Differences between categorical variables were tested with the $\chi^{2}$ test. Multiple logistic regression analysis was performed to identify independent correlates of $\triangle \mathrm{LAS}$ and to calculate HR with 95\% CI. Patient survival curves for the absence of adverse events (cardiovascular-mortality or heart failure hospitalisation) were constructed according to the KaplanMeier method and compared by the log-rank test. Sensitivity analysis was conducted using subgroup analysis and the leave-one-out approach. The statistical significance was defined as two-sided $p$ value $<0.05$. 
Table 1 Clinical characteristics of the study population

\begin{tabular}{|c|c|c|c|c|}
\hline & $\begin{array}{l}\text { All patients } \\
(n=100)\end{array}$ & $\begin{array}{l}\text { EVENTS } \\
(n=35)\end{array}$ & $\begin{array}{l}\text { NO EVENTS } \\
(n=65)\end{array}$ & $\begin{array}{l}\text { Significance } \\
\text { (p value) }\end{array}$ \\
\hline Age (years) & $81.2 \pm 5.3$ & $82 \pm 5.4$ & $80.7 \pm 5.3$ & 0.244 \\
\hline Female, n (\%) & 52 (52) & $16(46)$ & $36(55)$ & 0.170 \\
\hline Weight (kg) & $70.9 \pm 13.4$ & $72.1 \pm 13.1$ & $70.2 \pm 13.5$ & 0.505 \\
\hline Height $(\mathrm{cm})$ & $161.1 \pm 7.7$ & $162.7 \pm 8$ & $160.1 \pm 7.4$ & 0.110 \\
\hline $\mathrm{BSA}\left(\mathrm{m}^{2}\right)$ & $1.78 \pm 0.19$ & $1.8 \pm 0.19$ & $1.76 \pm 0.18$ & 0.329 \\
\hline $\mathrm{BMI}\left(\mathrm{kg} / \mathrm{m}^{2}\right)$ & $27.3 \pm 4.7$ & $27.2 \pm 4.4$ & $27.4 \pm 4.9$ & 0.854 \\
\hline TAPSE pre-TAVR (mm) & $22 \pm 2.6$ & $22.4 \pm 2.6$ & $21.7 \pm 2.5$ & 0.285 \\
\hline AVA baseline $\left(\mathrm{cm}^{2}\right)$ & $0.75 \pm 0.15$ & $0.74 \pm 0.12$ & $0.76 \pm 0.17$ & 0.498 \\
\hline AV mean gradient pre-TAVR $(\mathrm{mm} \mathrm{Hg})$ & $47.9 \pm 13.4$ & $47.8 \pm 12.8$ & $48.1 \pm 13.9$ & 0.920 \\
\hline AV peak gradient pre-TAVR (mm Hg) & $74.4 \pm 18.5$ & $74.7 \pm 18.5$ & $74.3 \pm 18.6$ & 0.912 \\
\hline LVEF pre-TAVR (\%) & $53.7 \pm 9.1$ & $54.0 \pm 9.2$ & $53.6 \pm 9.1$ & 0.848 \\
\hline LVEDD pre-TAVR (mm) & $50.2 \pm 6.2$ & $49.9 \pm 6$ & $50.4 \pm 6.3$ & 0.746 \\
\hline LVESD pre-TAVR (mm) & $33.8 \pm 7.2$ & $33.2 \pm 5.7$ & $34.1 \pm 8$ & 0.610 \\
\hline IVSd pre-TAVR (mm) & $12.9 \pm 1.6$ & $12.9 \pm 1.7$ & $12.9 \pm 1.6$ & 0.996 \\
\hline LAVi pre-TAVR $\left(\mathrm{mL} / \mathrm{m}^{2}\right)$ & $48.1 \pm 13.8$ & $48.5 \pm 14.1$ & $47.9 \pm 13.8$ & 0.865 \\
\hline Aortic regurgitation pre-TAVR, n (\%) & & & & 0.066 \\
\hline None & $18(18)$ & $5(14)$ & $13(20)$ & - \\
\hline Mild & $48(48)$ & $20(57)$ & $28(43)$ & - \\
\hline Mild-to-moderate & $34(34)$ & $10(29)$ & $24(37)$ & - \\
\hline PASP (mm Hg) & $39 \pm 11.1$ & $38.5 \pm 10.4$ & $39.3 \pm 11.6$ & 0.793 \\
\hline E/A & $0.72 \pm 0.3$ & $0.71 \pm 0.2$ & $0.72 \pm 0.4$ & 0.923 \\
\hline average $E / E^{\prime}$ & $13.5 \pm 3.3$ & $12.9 \pm 2.6$ & $13.9 \pm 3.5$ & 0.234 \\
\hline EuroSCORE II (\%) & $6.8 \pm 4.7$ & $7.1 \pm 4.6$ & $6.6 \pm 4.8$ & 0.644 \\
\hline Creatinine (mg/dL) & $1.2 \pm 0.9$ & $1.1 \pm 0.5$ & $1.3 \pm 1$ & 0.317 \\
\hline $\mathrm{GFR}(\mathrm{mL} / \mathrm{min})$ & $54.9 \pm 21.7$ & $58.1 \pm 24.1$ & $53 \pm 20.1$ & 0.279 \\
\hline Platelets & $206 \pm 60.2$ & $196.4 \pm 55.8$ & $212.3 \pm 62.7$ & 0.225 \\
\hline AV mean gradient post-TAVR $(\mathrm{mm} \mathrm{Hg})$ & $9.7 \pm 4.8$ & $8.6 \pm 3.8$ & $10.4 \pm 5.3$ & 0.088 \\
\hline LAVi post-TAVR $\left(\mathrm{mL} / \mathrm{m}^{2}\right)$ & $47.3 \pm 15.3$ & $50.4 \pm 17.4$ & $43.8 \pm 12.2$ & 0.281 \\
\hline LVEF post-TAVR (\%) & $53.4 \pm 8.8$ & $53.5 \pm 8.2$ & $53.4 \pm 9.3$ & 0.983 \\
\hline Average E/E' post-TAVR & $12.2 \pm 2.6$ & $12.4 \pm 2.7$ & $12 \pm 2.5$ & 0.731 \\
\hline Aortic regurgitation post-TAVR, $n(\%)$ & & & & 0.962 \\
\hline None & $37(37)$ & $12(34)$ & $25(38)$ & - \\
\hline Mild & $57(57)$ & $21(60)$ & $36(55)$ & - \\
\hline Mild-to-moderate & $6(6)$ & $2(6)$ & $4(6)$ & - \\
\hline
\end{tabular}

AV, aortic valve; AVA, aortic valve area; BMI, body mass index; BSA, body surface area; E/A, early-to-late diastolic flow ratio; E/E', E-to-mean E' ratio; GFR, glomerular filtration rate; IVSd, interventricular septal diameter diastolic; LAVi, left atrial volume index; LVEDD, left ventricular end-diastolic diameter; LVEF, left ventricular ejection fraction; LVESD, left ventricular end-systolic diameter; PASP, pulmonary artery systolic pressure; TAPSE, tricuspid annular plane excursion; TAVR, transcatheter aortic valve replacement.

Sample size calculation. Based on previous literature, we estimated a mean LAS of $15 \%$ in the study population and hypothesised a 20\%-25\% reduction after TAVR. From previous literature, we expected that $30 \%-35 \%$ of study patients would have met the main clinical (composite) endpoint. Thus, we calculated that 96 patients would have been required to detect a $20 \%$ difference in $\triangle \mathrm{LAS}$ between the two groups with a $90 \%$ power and an alpha level of
0.005. However, the sample size was adjusted to 100 patients to account for patients/data lost.

\section{RESULTS}

The baseline characteristics of the study population are summarised in tables 1 and 2. Mean aortic valve area was $0.75 \pm 0.15 \mathrm{~cm}^{2}$. Ten patients presented with low-flow 
Table 2 Baseline and procedural characteristics $(n=100)$

\begin{tabular}{|c|c|}
\hline Hypertension, n (\%) & $90(90)$ \\
\hline Smoking, n (\%) & $16(16)$ \\
\hline Dyslipidaemia, n (\%) & $66(66)$ \\
\hline Diabetes, n (\%) & $28(28)$ \\
\hline Heart failure, n (\%) & $14(14)$ \\
\hline CKD, n (\%) & $29(29)$ \\
\hline Dialysis, n (\%) & $3(3)$ \\
\hline COPD, n (\%) & $16(16)$ \\
\hline Prior stroke/TIA, n (\%) & $9(9)$ \\
\hline Prior PCI, n (\%) & 19 (19) \\
\hline Prior AMI, n (\%) & $8(8)$ \\
\hline Prior CABG, $\mathrm{n}(\%)$ & $2(2)$ \\
\hline Prior valvular surgery, n (\%) & $0(0)$ \\
\hline \multicolumn{2}{|l|}{ Type of prosthesis } \\
\hline CoreValve, n (\%) & $1(1)$ \\
\hline CoreValve Evolut R, n (\%) & $69(69)$ \\
\hline CoreValve Evolut Pro, n (\%) & $1(1)$ \\
\hline SAPIEN 3, n (\%) & $28(28)$ \\
\hline Portico, n (\%) & $1(1)$ \\
\hline \multicolumn{2}{|l|}{ Prosthesis Dimension } \\
\hline 23 mm, n (\%) & $18(18)$ \\
\hline $25 \mathrm{~mm}, \mathrm{n}(\%)$ & $1(1)$ \\
\hline $26 \mathrm{~mm}, \mathrm{n}(\%)$ & $39(39)$ \\
\hline $28 \mathrm{~mm}, \mathrm{n}(\%)$ & $1(1)$ \\
\hline $29 \mathrm{~mm}, \mathrm{n}(\%)$ & $28(28)$ \\
\hline 34 mm, n (\%) & $6(6)$ \\
\hline
\end{tabular}

AMI, acute myocardial infarction; CABG, coronary artery bypass graft; CKD, chronic kidney disease; COPD, chronic obstructive pulmonary disease; $\mathrm{PCI}$, percutaneous coronary intervention; TIA, transient ischaemic attack.

low-gradient AS patients (aortic valve area $0.73 \pm 0.14 \mathrm{~cm}^{2}$ ), having a lower baseline mean gradient $(26.2 \pm 3.8 \mathrm{~mm}$ $\mathrm{Hg}, \mathrm{p}<0.001)$ and a lower LVEF $(36.9 \% \pm 6.7 \%, \mathrm{p}<0.001)$ compared with the remaining patients with classical highgradient normal flow AS (table 3 ).

Of the patients included in the study, 48 out of 100 had concomitant mild aortic regurgitation (AR), while 34 out 100 had mild-to-moderate AR. Mean baseline LAVi and LAS were $48.1 \pm 13.8 \mathrm{~mL} / \mathrm{m}^{2}$ and $15.3 \% \pm 5.9 \%$, respectively. Ninety per cent of the study population had concomitant arterial hypertension, $91 \%$ were in class NYHA III and nearly one-third presented with diabetes mellitus (table 2). All patients were on optimal medical treatment.

\section{Reproducibility}

Measurement of LA strain showed a good reproducibility. Mean interobserver variation for LAS was $0.28 \pm 0.65$, with an intraclass coefficient of correlation of 0.97 (95\% CI
0.93 to $0.99 ; \mathrm{p}<0.001)$ and a correlation coefficient of $0.98(\mathrm{p}<0.001)$.

\section{Post-procedural results}

TAVR was performed with either the balloon expandable EDWARDS Sapien $3(\mathrm{n}=28)$ or the self-expandable CoreValve $(\mathrm{n}=1)$, CoreValve Evolut $\mathrm{R}(\mathrm{n}=69)$, CoreValve Evolut Pro $(n=1)$ or Portico valve $(n=1)$. A procedural success of TAVR was reached in all the included patients. No fatal periprocedural complications occurred in any of the study patients. Fifty-seven patients $(57 \%)$ had postTAVR mild AR, whereas six patients $(6 \%)$ had mild-tomoderate post-TAVR AR. Finally, 21\% of patients had conduction disturbances requiring post procedural pacemaker implantation.

The mean post-TAVR transvalvular aortic gradient was $9.7 \pm 4.8 \mathrm{~mm} \mathrm{Hg}$. No significant change in LVEF was found after TAVR to $53.4 \% \pm 8.8 \% \quad(p=0.125)$. LAVi was non-significantly reduced after TAVR to $37.2 \pm 14.5 \mathrm{~mL} /$ $\mathrm{m}^{2}(\mathrm{p}=0.112)$. LAS was slightly improved after TAVR, although this difference was not statistically significant (LAS post-TAVR $15.8 \% \pm 7.2 \%, \mathrm{p}=0.649$ ).

\section{Impact of LA function on clinical events}

During a median follow-up of 31 months, 35 patients $(35 \%)$ met the combined clinical endpoint of cardiovascular death or hospitalisation for heart failure. Secondary endpoints are reported in table 4.

A lower $\triangle \mathrm{LAS}$ was significantly associated with a higher incidence of the combined clinical endpoint ( $\mathrm{HR}=0.76$ $(0.67-0.86) ; \mathrm{p}<0.001)$ (figure 2). No significant difference was found for LVEF, E/E', PASP, LAVi pre-TAVR (table 1, figure 2). A multivariate analysis was then run including $\triangle \mathrm{LAS}$, EuroSCORE II and LVEF. Results of the multivariate logistic regression analysis displayed in figure 3 showed that $\triangle \mathrm{LAS}(\mathrm{HR}=0.80, \mathrm{p}<0.001)$ was an independent predictor of the combined endpoint.

At Kaplan-Maier analysis (figure 4), stratification of the study population based on $\triangle \mathrm{LAS}$ showed that those with a $\triangle$ LAS above its median value had a significantly better event-free survival compared with those below the median $(\mathrm{p}<0.001)$.

Patients presented with low-flow low-gradient AS had lower baseline and post-TAVR LA strain, and lower $\triangle \mathrm{LAS}$ by comparison to the remaining patients with classical high-gradient normal flow AS (table 4). However, the presence of low-flow low-gradient AS was not associated with a higher incidence of adverse events during the follow-up $(\mathrm{p}=0.727)$.

\section{Predictors of reduction of LA peak systolic strain after TAVR}

We explored the predictors of LA peak systolic strain after TAVR amidst clinical, baseline and relative changes of echocardiographic parameters. Multivariate regression analysis identified larger pre-procedural left ventricular end-systolic diameter (LVESD) (coeff $\beta=-0.357, \mathrm{p}=0.009$ ) and female gender (coeff $\beta=0.350, p=0.007$ ) as independent predictors of a lower $\triangle \mathrm{LAS}$ after TAVR. 
Table 3 Characteristics of patient with low flow/low gradient AS

\begin{tabular}{|c|c|c|c|}
\hline & Low-flow, low-gradient AS & Normal-flow, high-gradient & Significance \\
\hline & $n=10$ & $\mathrm{n}=90$ & ( $p$ value) \\
\hline AVA baseline $\left(\mathrm{cm}^{2}\right)$ & $0.73 \pm 0.14$ & $0.76 \pm 0.15$ & 0.571 \\
\hline AV mean gradient baseline $(\mathrm{mm} \mathrm{Hg})$ & $26.2 \pm 3.8$ & $50.3 \pm 11.7$ & $<0.001$ \\
\hline PASP baseline (mm Hg) & $\begin{array}{l}39.3 \pm 12.7 \\
\%\end{array}$ & $\begin{array}{l}39.5 \pm 11.8 \\
\%\end{array}$ & 0.966 \\
\hline LVEF baseline (\%) & $36.9 \pm 6.7$ & $55.4 \pm 7.3$ & $<0.001$ \\
\hline LVEDD baseline (mm) & $55.3 \pm 9.1$ & $49.8 \pm 5.4$ & 0.006 \\
\hline LVESD baseline $(\mathrm{mm})$ & $43.8 \pm 10.7$ & $32.6 \pm 6$ & $<0.001$ \\
\hline IVSd (mm) & $11.6 \pm 1.3$ & $13.1 \pm 1.6$ & 0.006 \\
\hline TAPSE baseline (mm) & $18.8 \pm 2.4$ & $22 \pm 2.6$ & 0.005 \\
\hline$E / A$ & $0.8 \pm 0.6$ & $0.7 \pm 0.3$ & 0.501 \\
\hline E/E' average & $15 \pm 6$ & $13.5 \pm 2.8$ & 0.234 \\
\hline EuroSCORE II baseline (\%) & $12.0 \pm 13.4$ & $5.1 \pm 3.4$ & $<0.001$ \\
\hline LAS, pre-TAVR & $10.6 \pm 4.8$ & $15.6 \pm 5.9$ & 0.011 \\
\hline$\triangle \mathrm{LAS}$ & $-1.08 \pm 4.46 \%$ & $0.36 \pm 4.95 \%$ & 0.407 \\
\hline LAVi, pre-TAVR (mL/m²) & $49.7 \pm 22.5$ & $48.1 \pm 13.1$ & 0.791 \\
\hline$\Delta \mathrm{LAVi}$ & $-8.1 \pm 15.7$ & $-11.6 \pm 17.4$ & 0.672 \\
\hline
\end{tabular}

AS, aortic stenosis; AV, aortic valve; AVA, aortic valve area; E/A, early-to-late diastolic flow ratio; E/E', E-to-mean E' ratio; IVSd, interventricular septal diameter diastolic; LAS, left atrial strain; LAVi, left atrial volume index; LVEDD, left ventricular end-diastolic diameter; LVEF, left ventricular ejection fraction; LVESD, left ventricular end-systolic diameter; PASP, pulmonary artery systolic pressure; TAPSE, tricuspid annular plane excursion; TAVR, transcatheter aortic valve replacement.

\section{DISCUSSION}

To the best of our knowledge, this is the first study demonstrating that post-procedural decrease in LA reservoir function, assessed by means of the peak systolic $\triangle \mathrm{LAS}$, can predict adverse events (composite endpoint of cardiovascular-mortality or heart failure hospitalisation) in a long-term follow-up of patients with AS undergoing TAVR.

LA volume has been recently employed as a diagnostic and prognostic tool in cardiovascular disease, including AS. ${ }^{2-6}$ Recent reports ${ }^{1318}$ demonstrated LA volume and LA function do not progress side-by-side, because usually LA dysfunction precedes LA dilatation. It is widely acknowledged $^{7813}$ that LA function has three components: reservoir, conduit and booster pump. The reservoir function coincides with the LV systole; the conduit function derives from the blood passage from the LA to the LV in early diastole; and then, the booster pump function coming in late diastolic phase, corresponds to the LA contraction. The LA strain, specifically the peak positive longitudinal LA strain at end-systole assessed via 2D STE, which expresses the LA reservoir function, is a promising tool to evaluate LA function with incremental clinical value over LA volume. ${ }^{7-14}$ Interestingly, LA reservoir and conduit function are related to LV filling pressures and they are associated with LA fibrosis and remodelling in patients with cardiovascular disease, including severe mitral regurgitation undergoing surgery. ${ }^{14}$ 28-31 Impairment of diastolic function, as a consequence of AS and of increased LV filling pressures, has already been described. ${ }^{18}$ Recent studies have shown LA peak systolic strain is impaired in patients with AS compared with healthy controls. ${ }^{18}$ Moreover, others ${ }^{19}{ }^{21}$ demonstrated that LA peak systolic strain was an independent predictor of outcome in severe AS. Successful removal of LV pressure afterload (as after TAVR) has been revealed to normalise LA size, ${ }^{32}$ and patients who fail to do so remain threatened by recurrent adverse event. ${ }^{32} \mathrm{~A}$ short-term improvement in reservoir LA function following TAVR, assessed by 2D STE, has been described in recent reports. ${ }^{20}$ In this context, our results confirm and extent previous literature also demonstrating that the lack of improvement in reservoir

Table 4 Secondary endpoints

\begin{tabular}{lllll}
\hline & All patients & High $\Delta$ LAS & Low $\Delta$ LAS & Significance (p value) \\
\hline All-cause death & 11 & 2 & 9 & 0.043 \\
Myocardial infarction & 0 & 0 & 0 & - \\
Stroke/transient ischaemic attack & 3 & 0 & 3 & 0.089 \\
\hline
\end{tabular}

LAS, left atrial strain. 
LA function following TAVR, measured by means of the $\triangle \mathrm{LAS}$, is associated with worse outcome in a long-term follow-up. In line with previous reports, our findings did show that $\triangle \mathrm{LAVi}$ was numerically higher in patients with events as compared with those who did not develop any clinical complications. However, this association was not statistically significant. This suggests that $\triangle \mathrm{LAS}$ is a more sensitive prognostic marker than $\triangle \mathrm{LAVi}$, particularly for patients being at an earlier pathophysiological stage of the disease, as those enrolled in our study. In fact, it has been previously demonstrated that LAS has a higher sensitivity to detect earlier LA dysfunction by comparison to LA volume. . $^{-6} 1318$

On the contrary, we found an association between the post-TAVI recovery/impairment of LA function, in the form of $\triangle \mathrm{LAS}$, and a pre-existing larger LVESD and the presence of a male gender. Therefore, the lack of functional improvement might be related at least in part to the LVESD before procedure and more frequent in male patients with AS than females. The latter result suggests that the well-known gender differences, including the degree of concentric remodelling, of diastolic dysfunction and of vascular stiffness, might exert a sensible impact on the response to treatment. ${ }^{33} 34$

Our results suggest that the short-term increase of $\triangle$ LAS after TAVR may be useful to identify a category of patients at higher risk of cardiovascular mortality and hospital readmission as the result of recurrent heart failure. In these patients, a more diligent clinical control and a more aggressive medical therapy may be advantageous to prevent adverse clinical events.

\section{Limitations}

This is a single centre study. However, changes observed in our relatively small cohort are consistent, and support our data interpretation. Poor acoustic windows may prevent the precise characterisation of LA myocardial deformation and lead to potential mistakes in epicardial/ endocardial border tracing. However, a careful endocardial delineation of LA myocardial segments, especially if performed by an experienced echocardiographer, and the exclusion from the study analysis of patients with poor image resolution, allow acquiring reproducible and reliable data.

\section{CONCLUSIONS}

This study demonstrated an improvement in LAS after TAVR and suggests it might be used as a prognostic marker. In fact, a lower reduction in $\triangle \mathrm{LAS}$ in patients after TAVR was an independent predictor of the composite endpoint of cardiovascular death and hospitalisation for heart failure. Larger studies are required to confirm the predictive power of $\triangle \mathrm{LAS}$ for patients' risk stratification after TAVR.

Contributors JS: Funding acquisition, Investigation, Methodology, Writing original draft; SDR: Writing—review and editing, Visualisation, Supervision; IL: Data curation, Investigation; AS: Data curation, Investigation; SLB: Data curation,
Investigation; SS: Software, Visualisation; AM: Investigation, Visualisation; CS: Investigation, Visualisation; AP: Data curation; Cl: Funding acquisition, Supervision, Writing - review and editing.

Funding The authors have not declared a specific grant for this research from any funding agency in the public, commercial or not-for-profit sectors.

Competing interests None declared.

Patient consent for publication Not required.

Ethics approval The study complies with the Declaration of Helsinki. The research protocol has been approved by the locally appointed ethics committee (Comitato Etico Regione Calabria-Area Centro) and informed consent has been obtained from participating subjects.

Provenance and peer review Not commissioned; externally peer reviewed.

Data availability statement De-identified participant data are available upon request from Dr Jolanda Sabatino (jolesbt@hotmail.it).

Open access This is an open access article distributed in accordance with the Creative Commons Attribution Non Commercial (CC BY-NC 4.0) license, which permits others to distribute, remix, adapt, build upon this work non-commercially, and license their derivative works on different terms, provided the original work is properly cited, appropriate credit is given, any changes made indicated, and the use is non-commercial. See: http://creativecommons.org/licenses/by-nc/4.0/.

ORCID iD

Salvatore De Rosa http://orcid.org/0000-0001-5388-942X

\section{REFERENCES}

1 Lancellotti P, Moonen M, Magne J, et al. Prognostic effect of longaxis left ventricular dysfunction and B-type natriuretic peptide levels in asymptomatic aortic stenosis. Am J Cardiol 2010;105:383-8.

2 Beach JM, Mihaljevic T, Rajeswaran J, et al. Ventricular hypertrophy and left atrial dilatation persist and are associated with reduced survival after valve replacement for aortic stenosis. J Thorac Cardiovasc Surg 2014;147:362-9.

3 Hoit BD. Left atrial size and function: role in prognosis. J Am Coll Cardiol 2014;63:493-505.

4 Moller JE, Hillis GS, Oh JK, et al. Left atrial volume: a powerful predictor of survival after acute myocardial infarction. Circulation 2003;107:2207-12.

5 Rossi A, Cicoira M, Zanolla L, et al. Determinants and prognostic value of left atrial volume in patients with dilated cardiomyopathy. $J$ Am Coll Cardiol 2002;40:1425-30.

6 Russo C, Jin Z, Homma S, et al. Left atrial minimum volume and reservoir function as correlates of left ventricular diastolic function: impact of left ventricular systolic function. Heart 2012;98:813-20.

7 Cameli M, Caputo M, Mondillo S, et al. Feasibility and reference values of left atrial longitudinal strain imaging by two-dimensional speckle tracking. Cardiovasc Ultrasound 2009;7:6.

8 Saraiva RM, Demirkol S, Buakhamsri A, et al. Left atrial strain measured by two-dimensional speckle tracking represents a new tool to evaluate left atrial function. J Am Soc Echocardiogr 2010;23:172-80.

9 D'Alto M, D'Andrea A, Di Salvo G, et al. Right atrial function and prognosis in idiopathic pulmonary arterial hypertension. Int $J$ Cardiol 2017;248:320-5.

10 Di Salvo G, Pacileo G, Castaldi B, et al. Two-dimensional strain and atrial function: a study on patients after percutaneous closure of atrial septal defect. Eur J Echocardiogr 2009;10:256-9.

11 Di Salvo G, Pacileo G, Del Giudice EM, et al. Atrial myocardial deformation properties in obese nonhypertensive children. J Am Soc Echocardiogr 2008;21:151-6.

12 Vianna-Pinton R, Moreno CA, Baxter CM, et al. Two-dimensional speckle-tracking echocardiography of the left atrium: feasibility and regional contraction and relaxation differences in normal subjects. $J$ Am Soc Echocardiogr 2009;22:299-305.

13 Mondillo S, Cameli M, Caputo ML, et al. Early detection of left atrial strain abnormalities by speckle-tracking in hypertensive and diabetic patients with normal left atrial size. J Am Soc Echocardiogr 2011;24:898-908.

14 Sabatino J, Di Salvo G, Prota C, et al. Left atrial strain to identify diastolic dysfunction in children with cardiomyopathies. J Clin Med 2019;8:1243.

15 Singh A, Addetia K, Maffessanti F, et al. LA Strain for Categorization of LV Diastolic Dysfunction. JACC Cardiovasc Imaging 2017;10:735-43. 
16 Schneider C, Malisius R, Krause K, et al. Strain rate imaging for functional quantification of the left atrium: atrial deformation predicts the maintenance of sinus rhythm after catheter ablation of atrial fibrillation. Eur Heart J 2008;29:1397-409.

17 Vasquez N, Ostrander BT, Lu D-Y, et al. Low left atrial strain is associated with adverse outcomes in hypertrophic cardiomyopathy patients. J Am Soc Echocardiogr 2019;32:593-603.

18 O'Connor K, Magne J, Rosca M, et al. Left atrial function and remodelling in aortic stenosis. Eur J Echocardiogr 2011;12:299-305.

19 Galli E, Fournet M, Chabanne C, et al. Prognostic value of left atrial reservoir function in patients with severe aortic stenosis: a 2D speckle-tracking echocardiographic study. Eur Heart J Cardiovasc Imaging 2016;17:533-41.

20 D'Ascenzi F, Cameli M, Henein M, et al. Left atrial remodelling in patients undergoing transcatheter aortic valve implantation: a speckle-tracking prospective, longitudinal study. Int J Cardiovasc Imaging 2013;29:1717-24.

21 Meimoun P, Djebali M, Botoro T, et al. Left atrial strain and distensibility in relation to left ventricular dysfunction and prognosis in aortic stenosis. Echocardiography 2019;36:469-77.

22 Indolfi C, Sabatino J, De Rosa S, et al. Description and validation of TAVIApp: a novel mobile application for support of physicians in the management of aortic Stenosis-Management of aortic stenosis with TAVIApp. Biomed Res Int 2017;2017:1-8.

23 Sabatino J, De Rosa S, Leo I, et al. Non-invasive myocardial work is reduced during transient acute coronary occlusion. PLoS One 2020;15:e0244397.

24 Baumgartner $\mathrm{H}$, Hung J, Bermejo J, et al. Recommendations on the echocardiographic assessment of aortic valve stenosis: a focused update from the European Association of Cardiovascular Imaging and the American Society of Echocardiography. J Am Soc Echocardiogr 2017;30:372-92.

25 Pibarot P, Clavel M-A. Left ventricular outflow tract geometry and dynamics in aortic stenosis: implications for the echocardiographic assessment of aortic valve area. J Am Soc Echocardiogr 2015;28:1267-9.
26 Lang RM, Badano LP, Mor-Avi V, et al. Recommendations for cardiac chamber quantification by echocardiography in adults: an update from the American Society of Echocardiography and the European Association of Cardiovascular Imaging. J Am Soc Echocardiogr 2015;28:1-39.

27 Nagueh SF, Smiseth OA, Appleton CP, et al. Recommendations for the evaluation of left ventricular diastolic function by echocardiography: an update from the American Society of Echocardiography and the European Association of Cardiovascular Imaging. Eur Heart J Cardiovasc Imaging 2016;17:1321-60.

28 Hsiao S-H, Chiou K-R, Lin K-L, et al. Left atrial distensibility and E/e' for estimating left ventricular filling pressure in patients with stable angina. -A comparative echocardiography and catheterization study-. Circ J 2011;75:1942-50.

29 Hsiao S-H, Huang W-C, Lin K-L, et al. Left atrial distensibility and left ventricular filling pressure in acute versus chronic severe mitral regurgitation. Am J Cardiol 2010;105:709-15.

30 Hsiao S-H, Chu K-A, Wu C-J, et al. Left atrial expansion index predicts left ventricular filling pressure and adverse events in acute heart failure with severe left ventricular dysfunction. J Card Fail 2016;22:272-9.

31 Hsiao S-H, Chiou K-R, Porter TR, et al. Left atrial parameters in the estimation of left ventricular filling pressure and prognosis in patients with acute coronary syndrome. Am J Cardiol 2011;107:1117-24.

32 De Rosa R, Murray M-I, Schranz D, et al. Short-term decrease of left atrial size predicts clinical outcome in patients with severe aortic stenosis undergoing TAVR. Catheter Cardiovasc Interv 2020;96:E341-7.

33 Espinola-Zavaleta N, Antonio-Villa NE, Briseño-Diaz N, et al. Left ventricular and atrial global strain evaluation within subtypes of ventricular remodeling. Echocardiography 2021;38:280-8.

34 Spaccarotella C, Mongiardo A, Indolfi C. Pathophysiology of aortic stenosis and approach to treatment with percutaneous valve implantation. Circ J 2011;75:11-19. 